\title{
A Three-Year Experience with GeneXpert MTB/RIF Assay in Tuberculosis Control Programme (RNTCP)- A Clinical Study
}

\author{
Santosh Kumar Nemagouda ${ }^{1}$ \\ ${ }^{1}$ Associate Professor, Department of Pulmonology, Sri B.M. Patil Medical College, BLDE University, \\ Vijayapur, Karnataka, India.
}

\section{ABSTRACT}

\section{BACKGROUND}

MDR-TB caused an estimated 600,000 new TB cases and 240,000 deaths in 2016 and MDR-TB accounts for $4.1 \%$ of all new TB cases and 19\% of previously treated cases worldwide. Globally, most MDR-TB cases occur in South America, Southern Africa, India, China, and the former Soviet Union. Rapid detection of drug resistance of M. tuberculosis and MDR-TB strains ensures effective treatment of TB patients and limits further development of resistance to additional drugs. The GeneXpert test is a molecular test for TB which diagnoses TB by detecting the presence of TB bacteria, as well as testing for resistance to the drug Rifampicin. In India, where this test is starting to be widely used, it is known as the CBNAAT. The full name of the CBNAAT test is the Cartridge Based Nucleic Acid Amplification Test. The objective of the study is to assess the performance of GeneXpert MTB/RIF as an initial diagnostic tool for the diagnosis of Mycobacterium tuberculosis and Rifampicin Resistance.

\section{METHODS}

The RNTCP programme is being strictly followed and carried out in Shri B.M. Patil Medical college Hospital, BLDE University Vijayapur Karnataka. Samples of patients with symptoms suggestive of tuberculosis including, suspected new TB cases, old TB cases and on treatment TB cases, were received from all the Primary Healthcare Canters, and all other Healthcare Centres including two Medical College Hospitals of Vijayapur district. Samples collected under this programme were analysed by GeneXpert Test at District TB Hospital CBNAAT center Vijayapur, Karnataka. A retrospective study was conducted from 1 January 2016 to 31 December 2018. The samples were subjected to GeneXpert MTB/ RIF manufactured by Cepheid, France for the detection of M. tuberculosis and then rifampicin resistance in them. Data analysis was done using SPSS Version 22.0.0.0.

\section{RESULTS}

In this study a total of 4978 patients' samples was processed for CBNAAT test. Out of these cases, MTB was detected in 1186 (23.82\%), Rifampicin Resistance (RR) was detected in 165 (13.91\%) cases. Among these 165 MTB-RR cases, the MTB detection level was, 50 (30.30\%) High, 65 (39.39\%) Medium, 26 (15.79\%) Low, 16 (9.69\%) Very Low \& 8 (4.84\%) Intermediate. Out of 4978 cases 427 (8.58\%) were HIV positive, among these HIV positive cases MTB was detected in $51(12 \%)$ \& RR detected in 13 (25.50\%) cases.

\section{CONCLUSIONS}

The implementation of the GeneXpert MTB/RIF assay in programmatic management of TB \& MDR-TB in RNTCP has dramatically improved the rapid diagnosis of tuberculosis \& drug resistance, especially in People Living with HIV/AIDS (PLWHA).

\section{KEY WORDS}

RNTCP, CBNAAT, GeneXpert Test, Xpert MTB/RIF, HIV/AIDS
Corresponding Author: Dr. Santosh Kumar Nemagouda, S/o. R. S. Nemagouda, Sri Siddeshwar Housing Colony, \#85, North Athani Road, Vijayapur, Karnataka, India.

E-mail: pulmonologist2007@yahoo.co.in

DOI: $10.14260 /$ jemds $/ 2019 / 669$

Financial or Other Competing Interests: None.

How to Cite This Article:

Nemagouda SK. A three-year experience with genexpert MTB/RIF assay in tuberculosis control programme (RNTCP)a clinical study. J. Evolution Med. Dent. Sci. 2019;8(41):3080-3083, DOI: 10.14260/jemds/2019/669

Submission 19-08-2019, Peer Review 28-09-2019, Acceptance 04-10-2019, Published 14-10-2019. 


\section{BACKGROUND}

Tuberculosis (TB) is an ancient disease of mankind. TB is an infectious and contagious disease. TB is caused by Mycobacterium tuberculosis (MT). MT has very ancient origins; it has survived over 70,000 years and it currently infects nearly 2 billion people worldwide,[1] with around 10.4 million new cases of TB each year, almost one third of the world's population are carriers of the TB bacillus and are at risk for developing active disease.[2] TB has always been associated with a high mortality rate over the centuries, and also nowadays, it is estimated to be responsible for 1.4 million TB deaths, among infectious diseases after human immunodeficiency virus (HIV).[2] In India the prevalence of TB is 299 cases per 100000 population or 3.4 million prevalent cases.[3]

In the year 1961 government of India had launched National TB Programme (NTP). The shortcomings of the NTP were highlighted by the Joint Review by the Government of India, the Swedish International Development Agency and WHO in 1992. The Revised National TB Control Programme (RNTCP) was established in 1993 and the programme is based on DOTS (Directly Observed Treatment Short course) the internationally-recommended strategy to control TB, was launched in 1997.[4]

Worldwide, sputum is most common and easily available respiratory specimen for the diagnosis of pulmonary TB and every guideline recommends the use of sputum-based tests. Recently, the World Health Organization (WHO) endorsed a new, rapid, automated, 2-hour molecular test called Xpert MTB/RIF, based on the GeneXpert platform (Cepheid Inc, USA) which can diagnose TB with great accuracy and can also detect those with drug resistance.[5] A recent Cochrane review has shown that the Xpert MTB/RIF test has $88 \%$ sensitivity and $98 \%$ specificity when compared to culture.[6] Xpert MTB/RIF can detect rifampicin resistance with a sensitivity of $94 \%$ and specificity of $98 \%$. Data from many countries, including India, clearly show substantially better performance of the Xpert MTB/RIF test over conventional smear microscopy.[6]

MDR-TB caused an estimated 600,000 new TB cases and 240,000 deaths in 2016 and MDR-TB accounts for $4.1 \%$ of all new TB cases and $19 \%$ of previously treated cases worldwide. Globally, most MDR-TB cases occur in South America, Southern Africa, India, China, and the former Soviet Union. Rapid detection of drug resistance of $M$. tuberculosis and MDR-TB strains ensures effective treatment of TB patients and limits further development of resistance to additional drugs. Hence, we studied the performance of CBNAAT in diagnosis TB and early detection of Rifampicin resistance in TB control programme (RNTCP).

\section{METHODS}

The RNTCP programme is being strictly followed and carried out in Shri BM Patil Medical college Hospital, BLDE University Vijayapur Karnataka. The samples of the patients with symptoms suggestive of Tuberculosis including, suspected new TB cases, old TB cases and on treatment TB cases, were received from all the Primary Healthcare Canters, and all other Healthcare Centres including two Medical College Hospitals of Vijayapur district. The samples were analysed by GeneXpert Test at District TB Hospital CBNAAT center Vijayapur, Karnataka. A retrospective study was conducted from 1 January 2016 to 31 December 2018. The samples were subjected to GeneXpert MTB/ RIF manufactured by Cepheid, France for the detection of M. tuberculosis and then rifampicin resistance in them. GeneXpert MTB/RIF is a cartridge-based nucleic acid amplification technique which includes semi-quantitative, nested real-time PCR in vitro diagnostic test for the detection of MTBC DNA in sputum samples or concentrated sediments prepared from induced or expectorated sputum. RIF-resistance associated mutations of the rpoB gene in the samples from patients at risk for rifampicin resistance. As per manufacturer's guidelines, $2 \mathrm{ml}$ of sample reagent is added to $1 \mathrm{ml}$ of each fresh sample directly into collection container. The lid was replaced and shaken vigorously 10-20 times. It was incubated at room temperature. After 10 minutes of incubation, the specimen was shaken vigorously 10-20 times. It was incubated for 5 minutes again. The sample was perfectly fluid before being processed with no more clumps of sputum. If still it is viscous, waited for 5-10 minutes further before processing it in the cartridge. At least $2 \mathrm{ml}$ of processed sample was taken with plastic transfer pipette from the collection container to single use, disposable, self-contained GeneXpert cartridge. Then, it was subjected to GeneXpert MTB/RIF to create a test. After scanning the cartridge barcode, loading was done on blinking module. The results were visualised on the attached computer and interpreted by using software. The GeneXpert MTB/RIF was repeated on the second sample if it has shown indeterminate susceptibility to RIF.

\section{Statistical Analysis}

Data analysis was done using SPSS Version 22.

\section{RESULTS}

In this study total of 4978 patient's samples were processed for CBNAAT test to assess the performance of GeneXpert/RIF Assay. Out of these 4978 cases MTB was detected in 1186 (23.82\%) cases and MTB was not detected in 3461 (69.52\%) cases, Error in interpretation were 159(3.19\%) cases, Invalid tests were $88(1.76 \%)$ cases and no results were found in $84(1.68 \%)$ cases [Table 1]. Among 1186 MTB detected cases, 1021 (86.09\%) cases were having Rifampicin sensitive TB and Rifampicin Resistance TB was detected in 165 (13.91\%) cases [Table 2]. Out of these 165 MTB-RR TB cases, the MTB detection level was, 50(30.30\%) High, 65(39.39\%) Medium, 26 (15.79\%) Low, 16(9.69\%) Very Low \& 8 (4.84\%) Intermediate [Table 3]. The CBNAAT, MTB detection levels in 1021 Rifampicin sensitive TB cases were 300 (29.38\%) High, 403 (39.47\%) Medium, 230(22.52\%) Low and 88(8.61\%) Very Low [Table 4].Out of 4978 cases 427 (8.58\%) were HIV positive, HIV negative cases were 2855(57.35\%) and HIV status was unknown in 1696 (34.06\%) cases [Table 5]. Out of these 427 HIV positive cases MTB was detected in 51 (12\%) cases and MTB was not detected in 376(88\%) cases [Table 6]. In 51 HIV-TB case, Rifampicin Resistant TB cases were 13 
(25.50\%) cases and Rifampicin sensitive TB case were 38 (74.50\%) [Table 7].

\begin{tabular}{|c|c|c|}
\hline Test Results & Absolute Number & Percentage \\
\hline MTB Detected & 1186 & $23.82 \%$ \\
\hline MTB Not Detected & 3461 & $69.52 \%$ \\
\hline Invalid Test & 88 & $1.76 \%$ \\
\hline Error during processing & 159 & $3.19 \%$ \\
\hline No Result & 84 & $1.68 \%$ \\
\hline \multicolumn{2}{|c|}{ Table 1. Total Number of Tests (4978) } \\
\hline
\end{tabular}

\begin{tabular}{|c|c|c|}
\hline Test Results & Absolute Number & Percentage \\
\hline RR-Not Detected & 1021 & $86.09 \%$ \\
\hline RR-Detected & 165 & $13.91 \%$ \\
\hline \multicolumn{2}{|c|}{ Table 2. MTB Detected Tests (1186) } \\
\hline
\end{tabular}

\begin{tabular}{|c|c|c|}
\hline MTB Detection Level & Absolute Number & Percentage \\
\hline High level & 50 & $30.30 \%$ \\
\hline Medium level & 65 & $39.39 \%$ \\
\hline Low level & 26 & $15.75 \%$ \\
\hline Very Low level & 16 & $09.69 \%$ \\
\hline Intermediate level & 08 & $04.84 \%$ \\
\hline \multicolumn{2}{|c|}{ Table 3. Rifampicin Resistant MTB Detection (165) }
\end{tabular}

\begin{tabular}{|c|c|c|}
\hline MTB Detection Level & Absolute Number & Percentage \\
\hline High level & 300 & $29.38 \%$ \\
\hline Medium level & 403 & $39.47 \%$ \\
\hline Low level & 230 & $22.52 \%$ \\
\hline Very Low level & 88 & $08.61 \%$ \\
\hline \multicolumn{2}{|c|}{ Table 4. Rifampicin Sensitive MTB Detection (1021) }
\end{tabular}

\begin{tabular}{|c|c|c|}
\hline Test Results & Absolute number & Percentage \\
\hline HIV Positive & 427 & $8.58 \%$ \\
\hline HIV Negative & 2855 & $57.35 \%$ \\
\hline Unknown & 1696 & $34.06 \%$ \\
\hline \multicolumn{3}{|c|}{ Table 5. HIV Status (4978) } \\
\hline
\end{tabular}

\begin{tabular}{|c|c|c|}
\hline Test Results & Absolute Number & Percentage \\
\hline MTB Detected & 51 & $12 \%$ \\
\hline MTB Not Detected & 376 & $88 \%$ \\
\hline \multicolumn{2}{|c|}{ Table 6. CBNAAT IN HIV Patients. (427) } \\
\hline
\end{tabular}

\begin{tabular}{|c|c|c|}
\hline Test Results & Absolute Number & Percentage \\
\hline RR- Detected & 13 & $25.50 \%$ \\
\hline RR-Not Detected & 38 & $74.50 \%$ \\
\hline \multicolumn{2}{|c|}{ Table 7. Rifampicin Resistance in HIV-TB Patients (51) } \\
\hline
\end{tabular}

\section{DISCUSSION}

In this study total of 4978 patient's samples were processed for CBNAAT test. Out of these cases MTB was detected in $1186(23.82 \%)$ cases. Out of these 1186 MTB detected cases Rifampicin sensitive TB detected in 1021 (86.08\%) cases and Rifampicin Resistance detected in 165 (13.91\%) cases. Similar results were found in Dravid MN et al. study, ${ }^{[7]}$ in their study out of total 475 samples MTB was detected in 193 (40.63\%). Out of these 193 MTB detected cases 160 (82.90\%) were sensitive to rifampicin and $33(17.10 \%)$ were resistance to rifampicin.

In Dravid MN et al. study, out of 193 MTB detected cases, 21(10.88\%) were HIV positive and 172 (89.12\%) were HIV negative. Out of the 21 HIV positive cases, $85.71 \%$ were rifampicin sensitive and $14.29 \%$ were Rifampicin resistant. [7] In present study, out of 4978 cases 427 (8.58\%) were HIV positive and among these HIV positive cases MTB detected in $51(12 \%)$ case. Out of HIV-TB, 38(74.50\%) were Sensitive TB cases and Rifampicin Resistance detected in 13 (25.50\%) cases. Sethi et al. reported HIV-TB co-infection in $20.1 \%$ cases $^{[8]}$ and in the study of Arora et al., $2.5 \%$ were HIV positive.[9]

According to WHO reports on MDR, MDR-TB in India continues to be reported in between $2.5-2.8 \%$ and $14-17 \%$ amongst new TB and retreated patients, respectively. ${ }^{[10-13]}$ In present study, the cases with Rifampicin resistance were 165 (13.91\%) among Old and New TB cases (1186).

The GeneXpert is an automated system, there is error in processing the samples, hence the cause of error may be too viscous sample (In this error code is 2008). The other causes of error could be probe check failure; incorrect volume of sample (Code error 5006, 5007) and heater failure can also cause error. In our study, error was detected in 159 (3.19\%) cases. In Bajrami et al. study the error was seen in $2.6 \%$ of cases.[14]

In present study out of 165 cases of rifampicin resistant TB, the MTB detection levels were 50 (30.30\%) High, 65 (39.39\%) Medium, 26 (15.79\%) Low, 16 (9.69\%) Very Low \& 8 (4.84\%) Intermediate. Out of 1021 Rifampicin sensitive TB cases the MTB detection levels were 300 (29.38\%) High, 403 (39.47) Medium, 230(22.52\%) Low and 88 (8.61\%) Very Low.

The Singh et al. study observations were, in a patient care setting in a high burden region, from a large collection of pulmonary and extra-pulmonary samples and puts to rest questions regarding sensitivity, specificity, detection of infrequent mutations and mutations responsible for low-level Rif resistance by GeneXpert. Improvements in the assay could offer further improvement in sensitivity of detection in different patient samples; nevertheless, it may be difficult to improve sensitivity of Rif resistance detection if only one gene is targeted. Assay specificity was high both for TB detection and Rif resistance detection. Despite a few misses, the assay offers major boost to early diagnosis of TB and MDR-TB, in difficult to diagnose pauci-bacillary TB.[15]

The Xpert MTB/RIF assay demonstrated a high capability to detect MTBC DNA in AFB microscopy- negative samples of pulmonary and extrapulmonary origin. With the exception of a specimen harbouring a mixed MTBC population, the assay correctly retrieved information from the rpoB hot spot region regarding RMP resistance. Accumulating published data, including those presented in Panayotis. Ioannidis et al., indicate that this rapid and easy-to-perform fully automated CBNAAT could prove to be an extremely helpful diagnostic tool in the fight against tuberculosis.[16]

García-Basteiro, A. L. et al. study Opined that the GeneXpert seems to be a reliable method for TB post-mortem diagnosis, more rapid and easy to perform than conventional molecular methods. And also shows that in high TB and HIV endemic countries, TB disease may be significantly underdiagnosed and often misdiagnosed. For individual and public health benefits, there is a need to develop and more widely apply new methods to reliably estimate TB mortality.[17]

Since children below 6 years are not able to expectorate the sputum, specimens like gastric lavage aspirate (GLA), induced sputum (IS), bronchoalveolar lavage (BAL) are used and each one will have its own advantages and disadvantages. In a study by Pang et al., the authors highlight limitations of their study and emphasize that Xpert MTB/RIF assay is not ideal for GA samples due to its low negative predictive value, which means overall low (70.1\%) 
specificity. They also emphasize that $\mathrm{NaHCO}_{3}$ neutralization of GA samples is not advisable if samples have to be stored and then cultured in MGIT960 system, though these samples may be used for DNA-based tests, as the DNA of dead bacilli can be amplified by later methods. They also showed that $11.6 \%$ Xpert MTB/Rif samples yielded indeterminant Rifampicin-resistance results, which is an important cost implication.[18]

Over the last decade, the RNTCP in India has expanded its TB services in phase wise manner to cover over 1.1 billion population. The RNTCP has met the WHO objectives for case detection and treatment success, and is close to fulfilling the TB-related Millennium Development Goal indicators and the Stop TB Partnership targets by 2015. The RNTCP has also proved that ensuring free TB services, like diagnosis, treatment, health awareness campaigns and research related activities via both the public and private sector is a costeffective and sound investment, providing a significant economic return to the country, and to the world. The RNTCP is doing well in the country; even though we have very poorly organised public health infrastructure and manpower by its commitment, careful planning, thorough implementation, stable funding. The use of an innovative network of technical consultants, has added in achieving the targets. In order to implement new activities related to the programme and to maintain the current programme activities, an annual amount of more than US\$100 million is required.

This level of expenditure on TB control activities is unprecedented in India; however the achievements of the RNTCP, with emphasis on the cost effectiveness of the programme, should help to convince both the Government of India and donors to increase their investment in the country with the world's greatest TB burden, both in terms of drugsusceptible and drug- resistant disease.

\section{CONCLUSIONS}

The implementation of the GeneXpert MTB/RIF assay in programmatic management of TB \& MDR-TB in RNTCP has dramatically improved the rapid diagnosis of tuberculosis \& drug resistance, especially in People Living with HIV/AIDS (PLWHA)

\section{REFERENCES}

[1] Mac Donald EM, Izzo AA. Tuberculosis vaccine development. In: Ribbon W, edr. Tuberculosis-expanding knowledge. In Tech 2015.

[2] Global Tuberculosis Report 2016. World Health Organization. [Accessed on 06/12/2016]. http://www.who.int/tb/publications/global_report/ en/

[3] Global Tuberculosis Control 2009: Epidemiology, Strategy, Financing. Geneva, World Health Organization, 2009.
[4] Raviglione MC, Pio A. Evolution of WHO policies for tuberculosis control, 1948-2001. Lancet 2002;359(9308): 775-80.

[5] Geneva: World Health Organization, 2011. World Health Organization. Policy statement: Automated real-time nucleic acid amplification technology for rapid and simultaneous detection of tuberculosis and rifampicin resistance: Xpert MTB/RIF system.

[6] Steingart KR, Sohn H, Schiller I, et al. Xpert® MTB/RIF assay for pulmonary tuberculosis and rifampicin resistance in adults. Cochrane Database Syst Rev 2013;(1):CD009593.

[7] Dravid MN, Shah P, Dange S, et al. Rapid detection of mycobacterium tuberculosis cases by GeneXpert- 1 year retrospective study. J Med Res Prac 2017;6(2):52-5.

[8] Sethi S, Mewara A, Dhatwalia SK, et al. Prevalence of multidrug resistance in mycobacterium tuberculosis isolates from HIV seropositive and seronegative patients with pulmonary tuberculosis in north India. BMC Infect Dis BioMed Central 2013;13:137.

[9] Arora D, Jindal N, Bansal R, et al. Rapid and accurate detection of mycobacterium tuberculosis in sputum samples by Cepheid Xpert assay-a clinical study. J Clin Diagn Res 2015;9(5):DC03-DC05.

[10] WHO report 2005: Global Tuberculosis control: surveillance, planning and financing. Geneva, World Health Organisation (WHO/HTM/TB/2005.349).

[11] WHO report 2006: Global Tuberculosis control, surveillance, planning and financing. Geneva, World Health Organisation (WHO/HTM/TB/2006.362).

[12] WHO report 2007: Global Tuberculosis control, surveillance, planning and financing. Geneva, World Health Organisation (WHO/HTM/TB/2007.376).

[13] WHO report 2008: Global Tuberculosis control, surveillance, planning and financing. Geneva, World Health Organisation (WHO/HTM/TB/2008.393).

[14] Bajrami R, Mulliqi G, Kurti A, et al. Comparison of GeneXpert MTB/RIF and conventional methods for the diagnosis of tuberculosis in Kosovo. J Infect Dev Ctries 2016;10(4):418-22.

[15] Singh UB, Pandey P, Mehta G, et al. Genotypic, phenotypic and clinical validation of GeneXpert in extra pulmonary and pulmonary tuberculosis in India. PLoS One 2016;11(2):e0149258.

[16] Ioannidis P, Papaventsis D, Karabela S, et al. Cepheid GeneXpert MTB/RIF assay for Mycobacterium tuberculosis detection and rifampin resistance identification in patients with substantial clinical indications of tuberculosis and smear-negative microscopy results. J Clin Microbiol 2011;49(8):3068-70.

[17] García-Basteiro AL, Ismail MR, Carrilho C, et al. The role of Xpert MTB/RIF in diagnosing pulmonary tuberculosis in post-mortem tissues. Sci Rep 2016;6:20703.

[18] Pang Y, Wang Y, Zhao S, et al. Evaluation of the Xpert MTB/RIF assay in gastric lavage aspirates for diagnosis of smear-negative childhood pulmonary tuberculosis. Pediatr Infect Dis J 2014;33(10):1047-51. 